\title{
Immunohistoochemical evaluation of GATA-3, pAKT and Ki-67 in triple negative breast carcinoma
}

\author{
Aryal Gopi ${ }^{1}$, Chhetri Aryal Sameer ${ }^{2}$, Soejima Yurie ${ }^{3}$, Sheckler Victoria ${ }^{4}$, Eishi \\ Yoshinobu ${ }^{3}$, Sawabe Motoji ${ }^{5}$ \\ ${ }^{I}$ Department of Pathology and Laboratory Medicine, Ashwins Medical College and Hospital, Nepal Mediciti Hospital Pvt. Ltd. \\ ${ }^{2}$ Department of Emergency Medicine, Alka Hospital Pvt. Ltd, Lalitpur, Nepal. \\ ${ }^{3}$ Department of Human Pathology, Graduate School of Medical and Dental Science, Tokyo Medical and Dental University, Japan. \\ ${ }^{4}$ Department of Science, Cornerstone University, Grand Rapids, MI USA. \\ ${ }^{5}$ Department of Molecular Pathology, Graduate School of Health Care Science, Tokyo Medical and Dental University, Japan.
}

\section{Keywords: \\ Breast; \\ Cancer; \\ GATA-3; \\ HER2-Neu; \\ pAKT; \\ Triple negative}

\begin{abstract}
Background: Triple-negative breast cancers lack expression of estrogen receptor, progesterone receptor, and human epidermal growth factor receptor -2 . These tumors have been known to be clinically aggressive. We evaluate GATA-3, pAKT and Ki-67 expression in 35 triple negative breast carcinomas.

Materials and Methods: A total of 35 triple negative breast carcinomas were included in this study. The histological subtyping was done according to Japanese guidelines for breast cancer.

Results: GATA-3 was positive in 60\% (21/35) of tumors. High GATA-3 expression was observed in ductal carcinoma in situ. pAKT expression was demonstrated in $85.7 \%(30 / 35)$ of TNBCs. The levels of expression of GATA-3 and pAKT showed no significant association with nuclear grading. $(\mathrm{P}=0.761$ and $\mathrm{P}=0.487$, respectively). Ki-67 labeling index was positively correlated with nuclear grading $(\mathrm{p}<0.001)$.

Conclusion: GATA-3 expression has no relation with ER and PR expression and pAKT and GATA-3 are strongly expressed in triple negative breast cancers.
\end{abstract}

\section{Correspondence:}

Dr. Gopi Aryal, MD, PhD

Chair, Department of Pathology and Laboratory Medicine, Ashwini Medical College and Hospital, Nepal Mediciti Hospital, Pvt. Ltd. ORCID ID: 0000-0002-8566-2593

Email: gopiaryal1@gmail.com

Reveived : June 6th, 2017 ; Accepted : August 9th, 2017; Published : September 1, 2017 Citation: Aryal G, Chhetri Aryal S, Soejima Y, Sheckler V, Eishi Y, Sawabe M. Immunohistoochemical evaluation of GATA-3, pAKT and Ki-67 in triple negative breast carcinoma. J Pathol Nepal. 2017;7:1196-201. doi: 10.3126/jpn.v7i2.18037

Copyright: This is an open-access article distributed under the terms of the Creative Commons Attribution 4.0 International License, which permits unrestricted use, distribution, and reproduction in any medium, provided the original author and source are credited.

\section{INTRODUCTION}

Breast cancer is a group of heterogeneous diseases that show substantial variation in their molecular and clinical characteristics. This heterogeneity poses significant challenges in studying the biology of the disease.

Triple-negative breast cancers (TNBC) lack expression of estrogen receptor (ER), progesterone receptor (PR), and human epidermal growth factor receptor -2 (HER-2/neu). TNBCs account for approximately $10-20 \%$ of all breast cancers. These tumors have been known to be clinically aggressive, and therapeutic options are limited because they are not amenable to hormone-based therapy or HER2 
Table 1: List of primary antibody information

\begin{tabular}{llllllll}
\hline $\begin{array}{l}\text { Primary } \\
\text { antibody }\end{array}$ & Clone & Source & Cat. \# & $\begin{array}{l}\text { Antigen } \\
\text { retrieval }\end{array}$ & Dilution & Isotype & Incubation condition \\
\hline ER & ID5 & $\begin{array}{l}\text { Diagnostic } \\
\text { bioSystem }\end{array}$ & Mob124 & WB/pH9 & $1: 50$ & Mouse IgG1, Kappa & RT for 30 minutes \\
PR & 16 & Leica & NCL-PGR-312 & AC/pH6 & $1: 100$ & Mouse IgG1 & RT for 30 minutes \\
HER2 & SV2-61y & $\begin{array}{l}\text { Nichirel } \\
\text { Bioscience Inc. }\end{array}$ & 427041 & Protease & Ready to use & Mouse & RT for 30 minutes \\
\hline Ki-67 & MIB-1 & Dako & M7240 & AC/pH9 & $1: 100$ & Mouse & RT for 30 minutes \\
\hline GATA-3-L & L50-823 & Cell Marque & $390 \mathrm{M}-16$ & WB/pH9 & $1: 200$ & Mouse, L50-823 & RT for 30 minutes \\
pAKT & (Ser473) & Cell signaling & P31749 & AC/pH9 & $1: 50$ & Rabbit IgG & Overnight at 40C
\end{tabular}

WB: water-bath $\left(95^{\circ} \mathrm{C}\right.$ for $\left.40 \mathrm{~min}\right)$

$A C$ : Autoclave $\left(121^{\circ} \mathrm{C}\right.$ for $\left.15 \mathrm{~min}\right)$

Protease (RT-room temperature for 5 minutes)

targeted therapy. A better understanding of the pathological mechanisms underlying TNBC development and progression may allow improved classification, risk stratification, and individualized treatment for this breast cancer subgroup. Recent gene profiling studies have identified at least 5 major subtypes of breast cancer, including normal breastlike, luminal A type, luminal B type, HER-2 positive type, and basal-like type. ${ }^{1}$

About $80 \%$ of TNBCs are known to share similar biologic characteristics with basal-like type breast cancer and is often accompanied by loss of functional BRCA-1. Basallike carcinomas are histologically characterized by a high Nottingham grade. It expresses basal-phenotype markers such as cytokeratin 5, 5/6, 15, 17 and EGFR. Based on patient demographic, association with BRCA-1 mutant and morphologic and immunohistochemical (IHC) features, it appears that basal-like breast carcinoma comprises a majority of medullary and atypical medullary carcinoma. However, medullary carcinoma is a rare subtype of basallike breast carcinoma with a fairly good prognosis.

GATA-binding protein 3 (GATA-3) is a member of the GATA family of zinc-finger binding transcription factor highly expressed by luminal epithelial cells in breast. It is involved in growth and differentiation of luminal epithelial cells. Gene-expression profiling has shown that GATA-3 is highly expressed in the luminal A subtype of breast cancer. ${ }^{2}$, ${ }^{3}$ Moreover, low GATA-3 expression is strongly associated with a higher histological grade, positive lymph nodes, ER and PR negative status, and HER2 overexpression, all of which are of poor prognosis. ${ }^{4}$

GATA-3 was positively correlated with ER and PR expression and inversely correlated with nuclear grade and Ki67 index. Expression of GATA-3 was associated with a better prognosis. ${ }^{5}$ In contrast, of 166 invasive breast carcinomas with 10-year follow-up information, there was no significant difference in survival rates between GATA-3positive and GATA-3-negative tumors. ${ }^{6}$
pAKT is a serine/threonine kinase which is fully activated when phosphorylated. pAKT plays a crucial role in cell survival and apoptosis. Downstream effects of pAKT activation include the phosphorylation and thereby inactivation of the protein BAD, a pro-apoptotic protein. ${ }^{7}$ Aberrant activation of pAKT is associated with breast carcinoma. pAkt was found to be positively associated with the lobular histological type. pAKT immunopositivity was inversely related to $\mathrm{Ki}-67$ and $\mathrm{p} 53 .{ }^{8}$ There was a significant association between both high cytoplasmic and nuclear pAKT expression with HER2 overexpression. ${ }^{8}$

Numerous studies have been published regarding proliferating activity of breast carcinoma. High labelling index of Ki-67 signifies poor prognostic factors. The aim of present study was to evaluate GATA-3, pAKT and Ki67 immunoreactivity in TNBCs. These were correlated with tumor nuclear grade.

\section{MATERIAL AND METHODS}

This study was approved by the institutional review board of Tokyo Medical and Dental University. A total of 35 triple negative breast carcinomas (ER-/PR-/HER2-), diagnosed at Tokyo Medical and Dental University Hospital by needle and operative biopsies from February 2010 to June 2011, were included in this study. The histological subtyping was done according to Japanese guidelines for breast cancer.

All specimens were fixed in 10\% formalin and embedded in paraffin. Formalin-fixed, paraffin embedded tissue was cut at a thickness of $4 \mathrm{um}$ and stained with Hematoxylin and Eosin stain.

For IHC, the sections were deparaffinized with xylene and rehydrated with graded ethanol. IHC was conducted using monoclonal and polyclonal antibodies and antigen-retrieval methods as listed in Table 1. After antigen retrieval, endogenous peroxidase was quenched with $3 \% \mathrm{H}^{2} \mathrm{O}^{2}$ in distilled water for $5 \mathrm{~min}$. This was followed by incubation with primary antibodies and the sections were stained by a detection method using En Vision (Dako) according to the manufacturer's protocol and counterstained with 
Table 2: Immunohistochemistry findings of TNBCs

\begin{tabular}{llll}
\hline Histological subtype & GATA-3 + & Source & Cat. \# \\
\hline No. $(\%)$ of positive cases & pAKT + & Diagnostic bioSystem & Mob124 \\
\hline No. $(\%)$ of positive cases & Ki-67 LI (\%) & Leica & NCL-PGR-312 \\
\hline DCIS & $4 / 4(100)$ & $3 / 4(75)$ & $10-20 \%$ \\
\hline IDC, NOS & $6 / 13(46.1)$ & $11 / 13(84.6)$ & $10-60 \%$ \\
PT & $5 / 9(55.6)$ & $8 / 9(88.9)$ & $10-90 \%$ \\
\hline ST & $4 / 6(66.7)$ & $6 / 6(100)$ & $30-90 \%$ \\
\hline Lobular carcinoma & $1 / 1(100)$ & $1 / 1(100)$ & $90 \%$ \\
Apocrine carcinoma & $1 / 2(50)$ & $1 / 2(50)$ & $10-60 \%$
\end{tabular}

DCIS: ductal carcinoma in situ; IDC-NOS: invasive ductal carcinoma, not otherwise specified; PT: papillotubular; ST: solid tubular

Table 3: Expression of GATA-3 in TNBCs

\begin{tabular}{|c|c|c|c|c|c|c|}
\hline & & Negative & Positive & & & Total \\
\hline & & 0 & $2+$ & $3+$ & $4+$ & \\
\hline \multirow[t]{3}{*}{ NG } & G1 & 4 & 3 & 3 & 2 & 12 \\
\hline & $\mathrm{G} 2$ & 5 & 2 & 1 & 2 & 10 \\
\hline & G3 & 5 & & 6 & 2 & 13 \\
\hline Total & & 14 & 5 & 10 & 6 & 35 \\
\hline
\end{tabular}

Hematoxylin. All antibodies were optimized and validated for maximum sensitivity and specificity.

Carcinomas were considered positive for ER and PR expression if positive staining of any intensity was present in $1 \%$ or more of nuclei. Carcinomas were considered positive for HER2 protein overexpression if invasive tumor cells showed $3+$ staining by IHC. ${ }^{10}$ The results of ER, PR and HER2 protein were obtained from archival database. In equivocal cases of ER, PR (5 cases in each) and HER2 protein (4 cases), repeat IHC was done for confirmation of negativity.

GATA3 labeling was scored on a scale of 0 to $4+$, with the extent of nuclear labeling graded as follows: $0(0-5 \%)$, $1+(6 \%-25 \%), 2+(26 \%-50 \%), 3+(51 \%-75 \%)$, and $4+$ $(>75 \%) .{ }^{11}$ We considered less than or equal to $5 \%$ staining as negative (score 0). Ductal epithelial cells in benign lobules were considered positive internal controls for GATA3 staining.

p-AKT was detected in the cytoplasm and the nuclei of the malignant cells. Staining intensity and the number of stained cells were taken into consideration throughout the evaluation process. The cytoplasmic and nuclear staining in tumor cells was scored as positive when moderate to strong staining was detected in more than $15 \%$ and $10 \%$ of the cells, respectively. In internal positive controls, pAKT was strongly expressed. Interpretation and scoring of K-67 labeling index was done according to the previously established guideline. ${ }^{12}$
Statistical analysis was performed using the statistical software SPSS version 19.0 (SPSS Inc., Chicago, IL). Correlation between markers was determined using Chi-square analysis. The results were considered to be statistically significant at $\mathrm{p}<0.05$.

\section{RESULTS}

Of the 35 TNBCs, there were 13 cases (37.1\%) of IDCNOS, 9 cases $(25.7 \%)$ of PT, 6 cases $(17.1 \%)$ of ST, 4 cases (11.4\%) of DCIS, 2 cases $(5.7 \%)$ of apocrine carcinoma and 1 case $(2.9 \%)$ of lobular carcinoma. The age of the patients ranged from 40 to 82 years. There were 32 cases of needle biopsies and 3 cases of operative biopsies. The IHC findings of this study are shown in Table 2 .

GATA-3 was positive in $60 \%(21 / 35)$ of TNBCs. The majority of GATA-3-positive cases (10/21, 47.5\%) showed a diffuse $(3+)$ nuclear staining pattern (fig. 1a\&b). High GATA-3 expression was observed in DCIS. The GATA-3 staining results for TNBCs are summarized in Table 3.

pAKT expression was demonstrated in $85.7 \%(30 / 35)$ of TNBCs, majority of them showing cytoplasmic expression. High pAKT expression was observed in ST subtype (Fig. $2 \mathrm{a}$ and $\mathrm{b}$ ). The pAKT immunostaining results of TNBCs are summarized in Table 4. The levels of expression of GATA-3 and $\mathrm{pAKT}$ showed no significant association with nuclear grading. ( $\mathrm{P}=0.761$ and $\mathrm{P}=0.487$, respectively).

There was no significant association between both high 


\begin{tabular}{|c|c|c|c|c|c|c|c|}
\hline & & Negative & Positive & & & & Total \\
\hline \multirow[t]{4}{*}{ Tumor type } & Grade & 0 & $1+$ & $2+$ & $3+$ & $4+$ & \\
\hline & G1 & 3 & 1 & 6 & 2 & & 12 \\
\hline & G2 & 1 & 1 & 3 & 4 & 1 & 10 \\
\hline & G3 & 1 & 3 & 4 & 5 & & 13 \\
\hline Total & & 5 & 5 & 13 & 11 & 1 & 35 \\
\hline
\end{tabular}
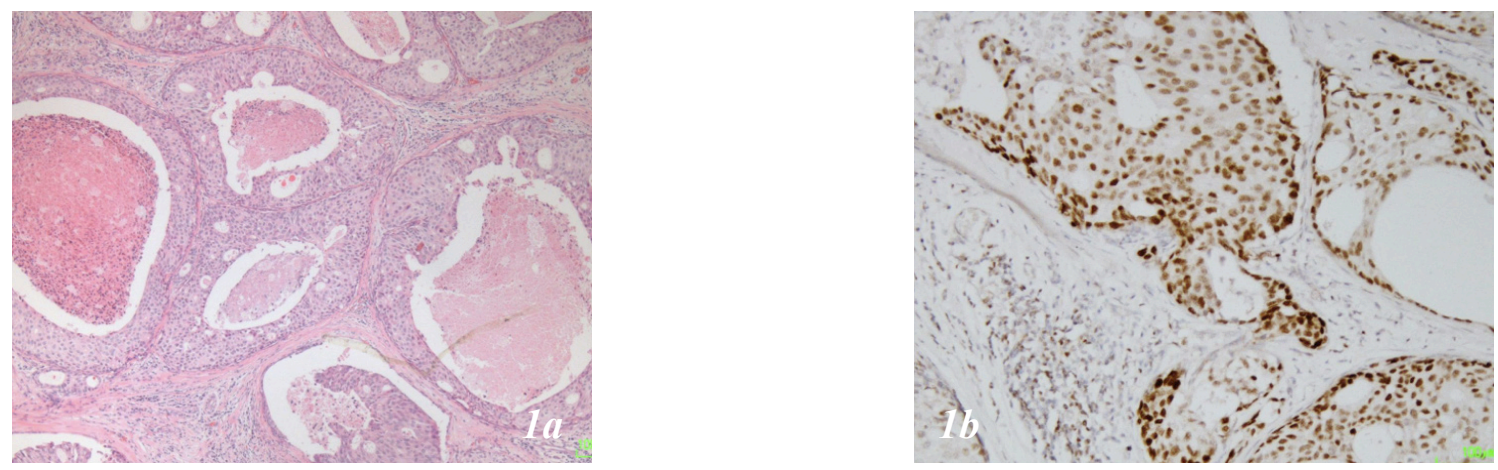

Figure 1a: Ductal carcinoma in situ, grade 1 (HE, x 100). b. GATA-3, 3+nuclear staining (x100)

cytoplasmic and nuclear pAKT expression with GATA-3 overexpression.

Ki-67 labelling index was positively correlated with nuclear grading $(\mathrm{p}<0.001$, Fig. 3a and 3b). Ki-67 index was variably expressed in $85.7 \%(30 / 35)$ of TNBC. The highest Ki-67 labelling index was observed in lobular carcinoma.

\section{DISCUSSION}

Due to the limited prognostic and predictive power of the existing classifications, since the beginning of the new century approaches have been considered to unveil the molecular basis for breast cancer heterogeneity. The breakthrough for breast cancer microarray studies occurred in 2000, when Perou and colleagues reported molecular classification of breast cancer using gene-expression analysis on DNA microarrays. ${ }^{2}$

ER-positive tumors are luminal tumors. A majority of these tumors (luminal A) are slow growing, have good prognosis and respond quite effectively to hormonal therapies. The remainder of ER-positive tumors is comparatively faster growing and still benefit from hormonal therapies, but a certain proportion may achieve huge benefit from additional chemotherapy. These tumors are termed luminal B type tumors. The other two molecular classification of breast cancers are HER2 enriched (HER2+, ER/PR-) and basal-like tumors (ER/PR-, HER 2-). This molecular classification has been shown to have prognostic value and to be predictive of the response to chemotherapy. ${ }^{13}$
The initia! attempts to extrapolate molecular findings to morphólogic and IHC criteria focused mainly on basal-like breast carcinoma. Morphologically, basal-like breast carcinomas are high-grade tumors with abundant lymphoplasmacytic infiltration and geographic necrosis.

GATA-3 is emerging as a sensitive and relatively specific immunomarkers for breast carcinomas. The expression of GATA-3 has a strong association with the expression of ER in breast cancer. ${ }^{14}$ GATA-3 expression is maintained in primary TNBCs and metastases of luminal cancers where ER expression is lost. This suggests that at least in some breast carcinomas, GATA-3 expression is dissociated from ER expression and signalling.

GATA-3+ tumors are correlated with lower grade, ER+, $\mathrm{PR}+$, and non-triple-negative phenotypes. ${ }^{15}$ However, male breast cancers showed no correlation between GATA-3 positivity and ER/PR positivity or distant metastases. ${ }^{16} \mathrm{~A}$ few reports in the literature have explored the expression of GATA-3 in TNBCs. Cimino-Mathews et al ${ }^{17}$ found that GATA-3 was expressed in $43 \%$ of TNBC. Liu $\mathrm{H}$ et al found that $69 \%(66 / 96)$ of ER-negative breast carcinomas expressed GATA-3). ${ }^{18}$ Our study revealed that $60 \%(21 / 35)$ of TNBCs expressed GATA-3. In summary, GATA-3 labelling is strongly expressed in both ER/PR-positive and Her-2-positive cases as wells as in TNBCs. The levels of expression of GATA-3 have no significant association with nuclear grading.

PTEN is a novel tumor suppressor gene located on 

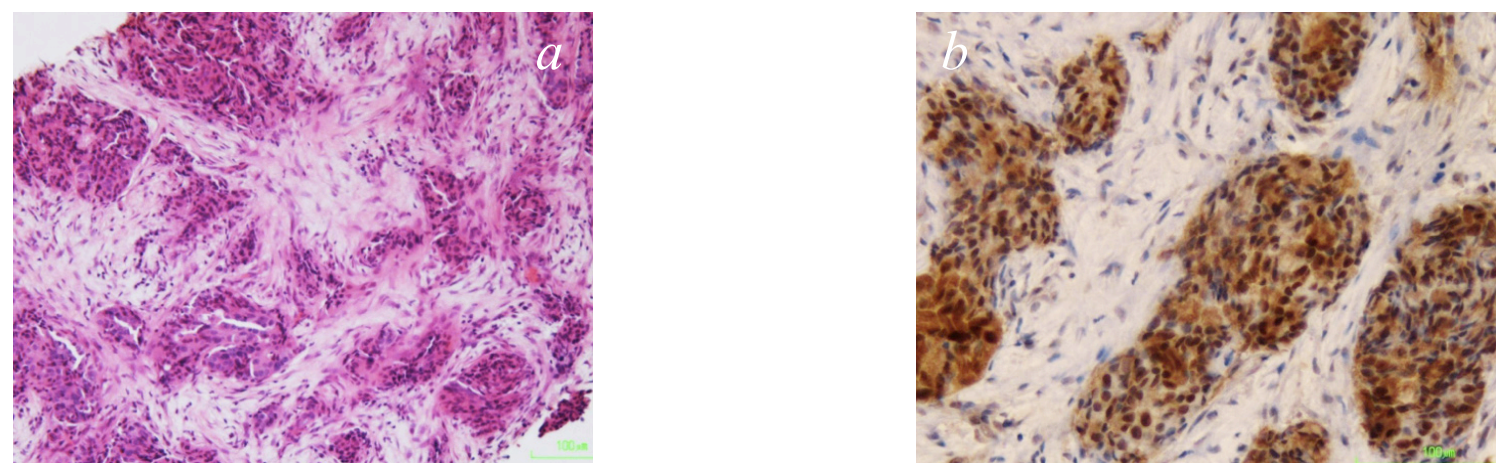

Figure 2: a. Invasive ductal carcinoma papillotubualr type, grade 3 (HE, x100). b. pAKT, 4+ nuclear staining (x100).
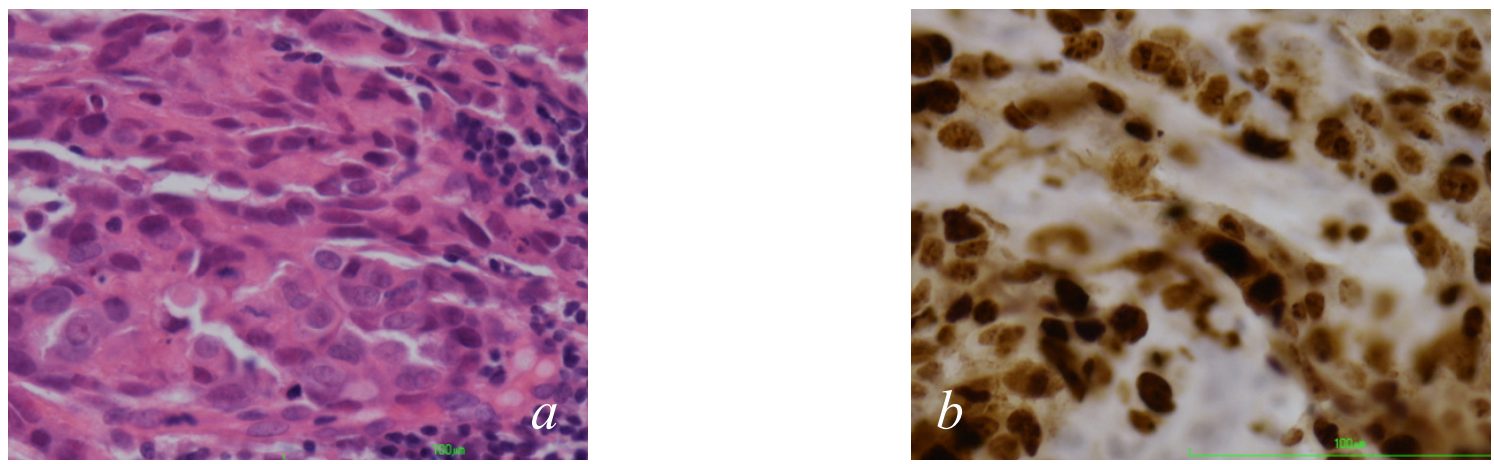

Figure3: a. Invasive ductal carcinoma, solid tubular, grade 3, (HE, x 400). b. Ki-67 labeling index 80\% (x400)

chromosome 10. Loss of PTEN leads to activation of AKT, which in turn promotes anti-apoptotic and pro-cell cycle entry pathways believed to be essential in tumourigenesis.

Seventy-six percent of the early invasive breast cancer overexpressed pAKT, where it was associated with expression of ER and androgen receptors. Higher proportions of luminal tumors were pAKT positive relative to $\mathrm{TNBCs} /$ basal subtypes. ${ }^{19}$

There is a positive correlation between pAKT and hormone receptors, which suggested the possible mechanism of endocrine resistance in ER-positive breast cancer.9 In our study, pAKT expression was demonstrated in $85.7 \%$ (30/35) of TNBCs. High pAKT expression was observed in the ST subtype.

\section{CONCLUSION}

From the study we can conclude that:

- GATA-3 expression has no relation with ER and PR expression

- pAKT and GATA-3 are strongly expressed in TNBCs

- The level of expression of GATA-3 and pAKT showed no significant association with nuclear grading

- pAKT, ? possible targeted therapy for TNBC in future.

\section{Conflict of Interest: None}

\section{REFERENCES}

1. Sorlie T, Tibshirani R, Parker J, et al. Repeated observation of breast tumor subtypes in independent gene expression data sets. Proc Natl Acad Sci . 2003;100:8418-23. Crossref

2. Perou CM, Sorlie T, Eisen MB, et al. Molecular portraits of human breast tumors. Nature. 2000;406:747-52. Crossref

3. Sorlie T, Perou CM, Tibshirani R, et al. Gene expression patterns of breast carcinomas distinguish tumor subclasses with clinical implications. Proc Natl Acad Sci 2001;98:10869-74. Crossref

4. Mehra R, Varambally S, Ding L, et al. Identification of GATA-3 as a breast cancer prognostic marker by global gene expression metaanalysis. Cancer Res. 2005;65:11259-64. Crossref

5. Hisamatsu Y, Tokunaga E, Yamashita N, et al. Impact of GATA-3 and FOXA1 expression in patients with hormone receptor-positive/ HER2-negative breast cancer. Breast Cancer. 2015;5:520-8. 
6. Ciocca V, Daskalakis C, Ciocca RM, Ruiz-Orrico A, Palazzo JP. The significance of GATA-3 expression in breast cancer: a 10-year follow-up study. Hum Pathol 2009;40:489-95. Crossref

7. Del Peso L, Gonza'lez-Garcı'a M, Page C, Herrera R, Nuñ ez G. Interleukin-3 induced phosphorylation of BAD through the protein kinase Akt.Science 278:687-9 Crossref

8. Magkou C, Mylona E, Theohari I, Giannopoulou I, Papanikolaou E, Markaki S, Nakopoulou L. An immunohistochemical evaluation of phosphorylated Akt at threonine 308 [pAkt(Thr308)] in invasive breast cancer. In Vivo. 2007;21:967-72. Crossref

9. Park SS, Kim SW. Activated Akt signaling pathway in invasive ductal carcinoma of the breast: correlation with HER2 overexpression. Oncol Rep. 2007;18:139-43. Crossref

10. Wolff AC, Hammond MEH, HICKS DG et al. Recommendations for Human Epidermal Growth Factor Receptor 2 Testing in Breast cancer. American Society of Clinical Oncology/College of American Pathologist. Clinical practice guideline update. J Clin Oncol 2013;31:3997-4014. Crossref

11. Yang M, Nonaka D. A study of immunohistochemical differential expression in pulmonary and mammary carcinomas. Mod Pathol. 2010;23:654-61. Crossref

12. Dowsett M, Nielsen TO, A'Hern R, et al. Assessment of Ki67 in breast cancer: recommendations from the International Ki67 in Breast Cancer working group. J Natl Cancer Inst. 2011;103:1656-64. Crossref
13. Rouzier R, Perou CM, Symmans WF et al. Breast cancer molecular subtypes respond differently to preoperative chemotherapy. Clin Cancer Res 2005;11:5678-85. Crossref

14. Fang SH1, Chen Y, Weigel RJ. GATA-3 as a marker of hormone response in breast cancer. J Surg Res. 2009;157:290-5. Crossref

15. Gulbahce HE1, Sweeney C, Surowiecka M, Knapp D, Varghese L, Blair CK. Significance of GATA-3expression in outcomes of patients with breast cancer who received systemic chemotherapy and/or hormonal therapy and clinicopathologic features of GATA-3-positive tumors. Hum Pathol. 2013;44:2427-31. Crossref

16. Gonzalez RS1, Wang J, Kraus T, Sullivan H, Adams AL, Cohen C.GATA-3expression in male and female breast cancers:comparison of clinicopathologic parameters and prognostic relevance.Hum Pathol 2013;44:1065-70. Crossref

17. Cimino-Mathews A, Subhawong AP, Illei PB et al. GATA-3 expression in breast carcinoma utility in triple negative, sarcomatoid, and metastatic carcinomas. Hum Pathol. 2013;44;1341-9. Crossref

18. Liu H1, Shi J, Prichard JW, Gong Y, Lin F. Immunohistochemical evaluation of GATA-3expression in ER-negative breast carcinomas. Am J Clin Pathol. 2014;141:648-55. Crossref

19. Aleskandarany MA, Rakha EA, Ahmed MA, Powe DG, Ellis IO, Green AR. Clinicopathologic and molecular significance of phosphoAkt expression in early invasive breast cancer. Breast Cancer Res Treat. 2011;127:407-16. Crossref 